\section{ITC 2/47}

Journal of Information Technology and Control

Vol. 47 / No. 2/ 2018

pp. 249-261

DOI 10.5755/j01.itc.47.2.18746

(C) Kaunas University of Technology
Two Faces of the Framework for Analysis and Prediction, Part 1 - Education
Received 2017/08/02

Accepted after revision 2018/03/07

\title{
Two Faces of the Framework for Analysis and Prediction, Part 1 - Education
}

\section{Vladimir Kurbalija, Mirjana Ivanović}

Department of Mathematics and Informatics, Faculty of Sciences, University of Novi Sad, Trg D. Obradovića 4, 21000 Novi Sad, Serbia, \{kurba,mira\}@dmi.uns.ac.rs

\section{Zoltan Geler}

Department of Media Studies, Faculty of Philosophy, University of Novi Sad, Dr Zorana Đinđića 2, 21000 Novi Sad, Serbia, zoltang@ff.uns.ac.rs

\section{Miloš Radovanović}

Department of Mathematics and Informatics, Faculty of Sciences, University of Novi Sad, Trg D. Obradovića 4, 21000 Novi Sad, Serbia, radacha@dmi.uns.ac.rs

Corresponding author: zoltang@ff.uns.ac.rs

With the ever-increasing amounts of data being generated in all walks of life and work, data analysis tools are gaining in importance and becoming essential in many application scenarios, including commerce, healthcare, research, and education. One important type of data are time series, which can be viewed as chronologically arranged arrays of numerical values that describe sequences of quantitative observations (e.g. in medical examinations, scientific and engineering experiments, etc.). In this article, we survey the successful applications of the Framework for Analysis and Prediction (FAP) - a Java-based tool dedicated to time-series analysis - in the area of education. Its utilization in this domain spans the development of several graphical user interfaces, as well as student projects illustrating and enhancing the capabilities of the system in the domains of time-series reconstruction, medicine (diagnosis), finance (stock value prediction) and psychology.

KEYWORDS: data mining; time-series analysis; tools for time-series processing; application of time-series in education. 


\section{Introduction}

Over the past two decades, due to the increasing need for processing ever-growing amounts of data, researching different aspects of data mining has gained more and more attention, becoming an important part of computer science and education [1]. Data mining can be described as the process of revealing and extracting potentially useful knowledge, interesting information, and novel patterns from vast quantities of data in order to support a wide range of decision making processes [12]. To accomplish this task, it combines solutions from several related research areas, such as machine learning, statistics, and database systems [29].

Temporal data mining is a sub-field of data mining that is focused on knowledge discovery from large collections of temporal data [25]. This form of data represents time-ordered sequences of numerical or categorical values. The most common type of temporal data are time series: they are composed of real values usually sampled at regular time intervals [11]. Such chronologically arranged arrays of numbers are used to express the change of the observed phenomena over time in a broad spectrum of different areas including finance, economics, medicine, engineering, meteorology, oceanography as well as in many other fields of natural and social sciences [2].

Statistical analysis of time series has a long history [15] and its main objectives are identifying patterns, trend analysis, seasonality and forecasting [4]. However, time-series data mining is concerned with tasks like indexing, classification, clustering, prediction, anomaly detection, data representation, and distance measures [7, 27]. According to Laxman and Sastry [22], there are some significant differences between statistical analysis and temporal data mining: data-mining applications must effectively analyze much larger volumes of data and their field of interest exceeds the scope of statistical time-series analysis.

The possibility of applying time series for storage, analysis, and visualization of data collected in many diverse domains led to a significant growth of interest in researching different task types of time-series data mining and resulted in a large amount of papers introducing new techniques and algorithms. However, alternative solutions are usually sporadically introduced in different publications and separately implemented, making it difficult to reproduce the presented results. Moreover, the newly-introduced methods have always claimed a particular superiority over some of the previous ones [27].

Taking these considerations into account, it is clear that the availability of a free and open sourced library could support and facilitate researching new and comparing existing techniques in this domain. Furthermore, it could be used as an auxiliary tool in research and education. Motivated by these deliberations, we have developed an extensible software package that implements many of the most important algorithms in the field of time-series data mining. Our framework facilitates numerous activities and applications of time series, including distance/ similarity measures, preprocessing, classification, and time-series representations. The basic concepts of our Framework for Analysis and Prediction (FAP) were presented in [20]. In this paper, we will give a detailed overview of the capabilities of FAP, and describe its applications in education realized at our institution, emphasizing the influence each application has had on the development of FAP. The companion paper, Two Faces of FAP, Part 2, will focus on applications in research.

The rest of this paper is organized as follows. Background knowledge on time series and distance measures is presented in the next section. Section 3 describes our Framework for Analysis and Prediction. Section 4 presents applications of the framework in education. Conclusions are given in Section 5.

\section{Time Series and Distance Measures}

A time series is a chronologically arranged array of numerical values that describes a sequence of quantitative observations [15]. Time series can represent results of medical examinations, scientific and engi- 
neering experiments, and they are suitable to characterize social, economic, and natural phenomena.

Formally, a $d$-dimensional multivariate time series $Q$ of length $n$ can be defined as a sequence of ordered pairs $Q=\left(q_{1}, t_{1}\right),\left(q_{2}, t_{2}\right), \ldots,\left(q_{n}, t_{n}\right)$, where $q_{i} \in R^{d}$ denotes the measured value of the observed phenomenon at timestamp $t_{i} \in R$ [1]. In this paper, we consider only univariate time series $(d=1)$ assuming that the measurements were performed at equidistant timestamps (i.e. $t_{i+1}=t_{i}+c, \forall i \in\{1, \ldots, n-1\}$ where $c$ is a constant value). In this manner, the time components of time series can be omitted, thus univariate time series $Q$ can be viewed as a sequence of $n$ real numbers: $\mathrm{Q}=\left(q_{1}, q_{2}, \ldots, q_{n}\right)[2]$.

Since similarity-based retrieval is one of the fundamental components of many time-series data mining tasks, the choice of an (in)appropriate measure is of crucial impact on their outcome [28]. However, unlike in the case of traditional databases, the similarity/distance ${ }^{1}$ between time series cannot be determined unambiguously. As a consequence, a great number of different approaches is proposed in the literature $[7,10,26]$. Among them, the two most frequently used time-series distance measures are Euclidean distance and Dynamic Time Warping (DTW) [9].

Euclidean distance represents a special case of Minkowski distance which is based on linear aligning of related points of time series: the $i$-th point of the first series is paired with the $i$-th point of the second one (Fig. 1a). Let $Q=\left(q_{1}, q_{2}, \ldots, q_{n}\right)$ and $S=\left(s_{1}, s_{2}, \ldots, s_{m}\right)$ denote two univariate time series of the same length $(n=m)$. Then, Euclidean distance is calculated as follows:

$$
d(Q, S)=\sqrt{\sum_{i=1}^{n}\left(q_{i}-s_{i}\right)^{2}} .
$$

Euclidean distance is a very simple measure and it has many advantages: it is easy to implement, fast to compute and represents a distance metric (allowing it to be used for indexing in time-series databases). However, it has some shortcomings, too: due to the linear aligning of the points, it is sensitive to distortions and shifting along the time axis [14].

To overcome this disadvantage, many different elastic distance measures were proposed: Dynamic Time Warping (DTW), Longest Common Subsequence (LCS), Edit Distance with Real Penalty (ERP), Edit Distance on Real sequence (EDR) [9], Time Warp Edit Distance (TWED) [23] and others. These distance measures rely on non-linear aligning of points - several points of one of the series can be paired with the same point of the other one, as illustrated in Fig. 1b. In the field of time-series data mining, one of the most popular elastic distance measures is DTW [30].

\section{Figure 1}

Aligning of time series: (a) linear; (b) non-linear

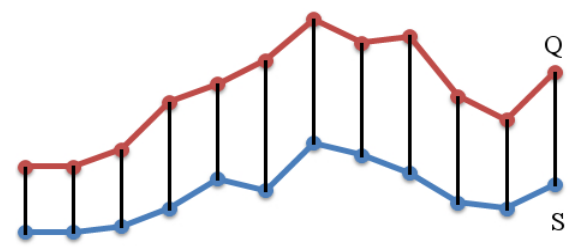

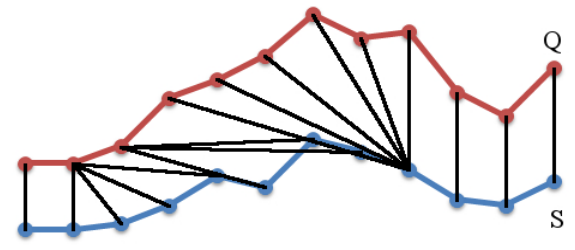

b

1 unless explicitly stated, we will not distinguish between similarity and distance measures, and will use the two terms interchangeably 
DTW defines the distance between two given time series $Q$ and $S$ of lengths $n$ and $m$ as the minimal accumulated distance between their points. This is achieved by searching for the optimal warping path (Fig. 2) in the warping matrix $\left[D_{i, j}\right]_{(n+1), m+1}$.

The optimal warping path which minimizes the warping distance between $Q$ and $S$ can be calculated using dynamic programming based on the following recursive definition:

$$
D_{i, j}=\left\{\begin{array}{cc}
0 & i=j=0, \\
\infty & i=0, j>0 \text { or } i>0, j=0, \\
d\left(q_{i}, s_{j}\right)+\min \left\{\begin{array}{l}
D_{i-1, j-1} \\
D_{i-1, j}
\end{array} \quad i, j \geq 1,\right. &
\end{array}\right.
$$

where $d\left(q_{i}, s_{j}\right)$ denotes the squared distance between $q_{i}$ and $s_{j}$. The distance between $Q$ and $S$ is then defined as $\operatorname{DTW}(\mathrm{Q}, S)=D_{n, m}$.

Figure 2

Optimal warping path inside the warping matrix

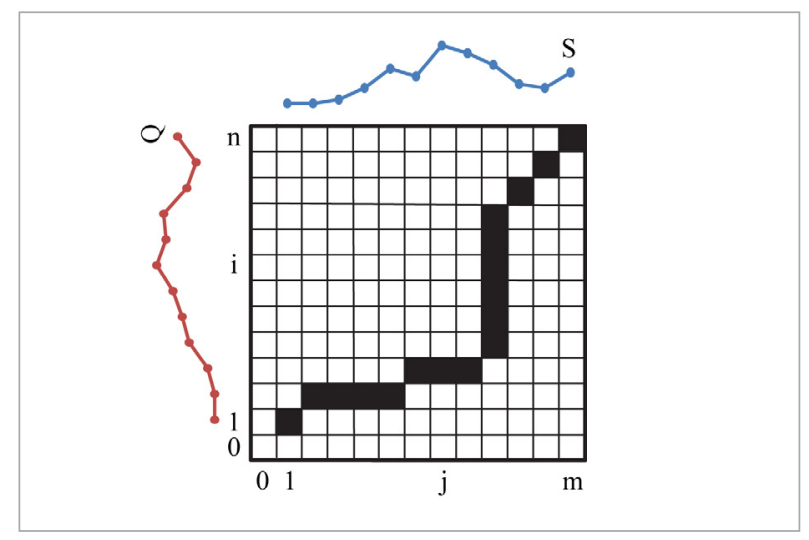

The quadratic computational complexity of finding the distance between time series can make the elastic measures not directly applicable to larger real-world problems. Furthermore, comparing each element of one time series with each element of the other one can lead to pathological aligning of the points (where a relatively small part of one time series maps onto a large section of the other time series). One way to avoid these shortcomings is to constrain the warping path using the Sakoe-Chiba band (Fig. 3a) or the Itakura parallelogram (Fig. 3b) [9].

\section{Figure 3}

Constraining the warping path using the: (a) Sakoe-Chiba band; (b) Itakura parallelogram

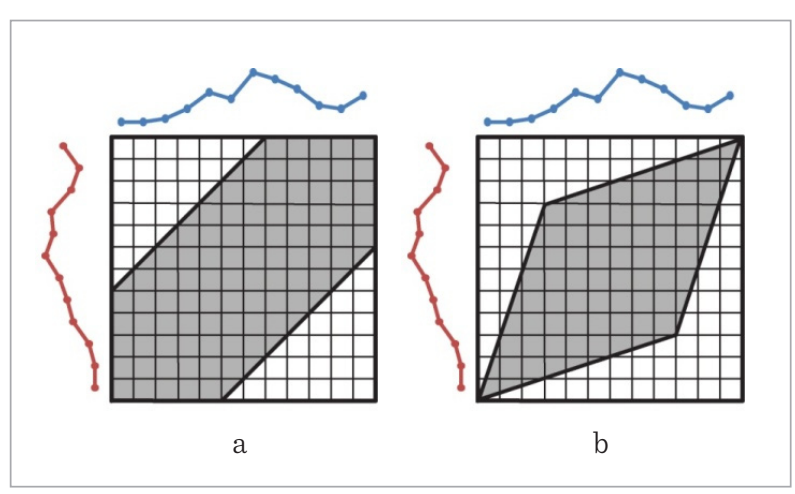

\section{Framework for Analysis and Prediction}

Framework for Analysis and Prediction (FAP http://perun.pmf.uns.ac.rs/fap/) is written in Java and designed to be a free and extensible open-source software package implementing all of the main techniques and methods for temporal data mining and analysis. It is developed and maintained at the Department of Mathematics and Informatics, Faculty of Sciences, University of Novi Sad, Serbia.

Overall architecture. The overall architecture of the framework is presented in Fig. 4a. The essential part of the library is implemented in the core package (Fig. 4b) which contains basic interfaces and classes that define the fundamental functionality of the system. In order to comply with the JavaBeans technology, all of the core classes support object serialization, have a default constructor and public getter and setter methods. The concrete implementations of the algorithms are placed in the appropriate subpackages of the library. In the rest of this section, we will summarize the fundamental interfaces and classes of the core package.

Time series. Time series objects are instances of the TimeSeries class from the fap.core.series package. They are implemented as series of data point objects. Data points are defined by the DataPoint class, and their series are represented by the DataPointSeries class.

In addition to the series of data points, every 
Figure 4

Overall architecture: (a) the FAP library; (b) the core package

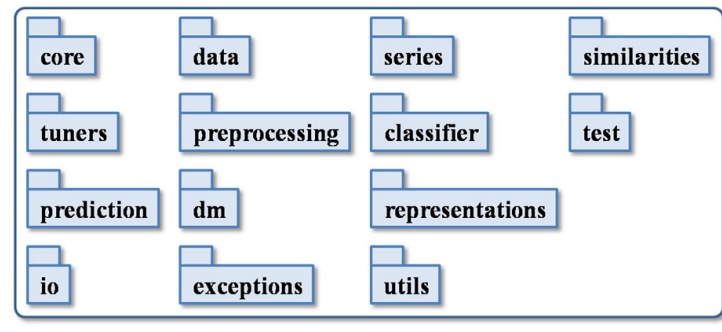

a

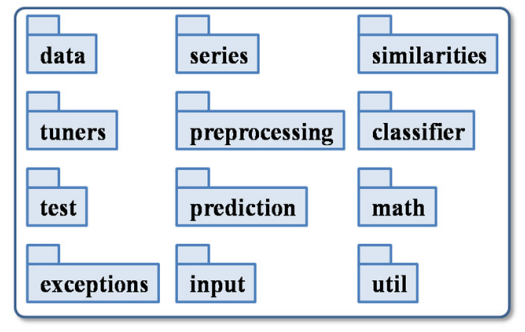

b
TimeSeries object contains a label (which represents the class of the time series), a supplementary property called index, and may have several representations. Time-series datasets are realized as objects of type TimeSeriesArrayList which extends the generic ArrayList class and defines several auxiliary methods.

Distance/similarity measures. Similarity measures represent essential ingredients of many time-series data mining tasks. Their role is to describe the similarity (or dissimilarity) between time series using numerical values. Classes that represent distance measures need to implement the SimilarityComputor interface which defines only one method that returns the distance between two given time series (Fig. 5a).

FAP contains implementations of several time-series distance/similarity measures, including: Minkowski distance $\left(\mathrm{L}_{\mathrm{p}}\right)$, Euclidean distance $\left(\mathrm{L}_{2}\right)$, Manhattan distance $\left(\mathrm{L}_{1}\right)$, Chebyshev distance $\left(\mathrm{L}_{\infty}\right)$, Canberra distance [5], Kulczynski distance [5], Lorentzian distance [5], Soergel distance [5], Sørensen distance [5], Spline distance [19], unconstrained and constrained DTW, LCS, ERP, EDR, and TWED. The constrained versions of the elastic measures are implemented using the Sakoe-Chiba band and the Itakura parallelogram [9].

Preprocessing. Sometimes we need to prepare and clean the raw data before using them, which is achieved by applying different techniques of data preprocessing. Our framework contains implementations of several preprocessing algorithms, including scaling of time-series length, shifting and z-score normalization, min-max normalization, and decimal scaling. Classes that represent preprocessing transformations of time series need to implement the
PreprocessingTransformation interface depicted in Fig. 5b.

Classification. Classification is the process of grouping objects into predefined categories, classes. It is done on the basis of a selected attribute (class label), which can have a finite number of different values. In this way, we always know the total number of different classes in advance.

The methods required for implementing classifiers are declared within the Classifier interface (Fig. 5c). The build method conducts training of the classifier based on the given dataset and similarity measure. The classify method is responsible for classifying the given time series. It should return the label selected by the classifying algorithm.

Our library contains the following classifiers: the simple nearest-neighbor classifier (1NN), the majority voting $k$-nearest neighbor classifier $(k \mathrm{NN})$, and the distance-weighted $k$-nearest neighbor classifier in combination with a wide variety of weighting schemes proposed in the literature, like Dudani's, Macleod's, the Fibonacci, the uniform and dual-uniform, Zavrel's, and the dual distance-weighted scheme [9].

The performance of a classifier can be measured by counting the number of correctly and incorrectly classified test objects. The accuracy of a classifier is defined as the ratio of test objects that are correctly classified, and the error rate is defined as the ratio of misclassified test objects. FAP implements the most popular partitioning techniques used to divide the initial set of labeled objects into training and test sets: holdout, $k$-fold (stratified) cross-validation, and leaveone-out [12]. In addition, auxiliary classes are provid- 
ed for performing repeated evaluations.

The basic methods for evaluating the performance of classifiers are declared within the Test interface (Fig. 5d). The test method is responsible for carrying out the evaluation process using the given dataset and the classifier set by the setClassifier method. The getErrorRatio method should return the average error ratio. The number of misclassified time series should be returned by the getMisclassified method.

Representations. Storing time series usually requires large amounts of space which makes performing different tasks of data mining more difficult. In addition, sometimes we are not interested in the exact values of each time-series data point. For these reasons, time-series databases generally contain only simplified representations of the series. Our library includes several representations, such as the Discrete Haar Wavelet Transform (Haar), Piecewise Linear Approximation (PLA), Piecewise Aggregate Approximation (PAA), Symbolic Aggregate Approximation (SAX) [17], Adaptive Piecewise Constant Approximation (APCA) [13], and Spline [19].

Classes that constitute representations of time series need to implement the TimeSeriesRepresentation interface which is presented in Fig. 5e. The getValue method should retrieve the value of the corresponding time series at the given value of the time component. The getOutboundValue method should return the value of time series outside of the range which is covered by current representation.

Resuming and tracking. FAP is designed to enable monitoring of long-time calculations through a callback mechanism, along with the possibility of their interruption without the loss of already obtained results. The incomplete computations can be resumed later. Tracking the execution of long-running processes is facilitated through the Callback (Fig. 5f), CallbackEnabled (Fig. 5g), and Resumable (Fig. 5h) interfaces. Combined with object serialization, they enable storing partial results of time-consuming tasks and the continuation of interrupted operations at a later time.

Methods that are necessary for the implementation of the callback mechanism are defined by the Callback interface. Classes that provide tracking of their activities should implement the CallbackEnabled interface and should regularly call the callback method of the appropriate Callback object in accordance with the configuration set through the getDesiredCallbackNumber and setPossibleCallbackNumber methods. The first of these two methods indicates how many times it is expected that they call the callback method. However, they do not have to comply with this expectation. Instead, they can themselves determine the number of callbacks based on their own needs and capabilities using the setPossibleCallbackNumber method.

Classes that perform long-running operations, and should support interrupting and resuming their execution, need to implement the Resumable interface. The reset method should reset the state of the objects and prepare them for reuse. In addition, these objects should indicate whether they have finished performing their task (isDone) and whether the execution is still in progress (isInProgress).

An example of using FAP (from the perspective of a computer science expert). To gain insight into the use of the FAP library, we will review the implementation of a repeated cross-validation experiment using our framework, as presented in Listing 1. After instantiating an NN classifier based on the Euclidean distance (line 1), we create a new 10-fold stratified cross-validation object (line 2). Then, in line 3, we use this object to get an instance of the RepeatedCrossValidation class with the given random seeds (line 5) which will be used for shuffling the dataset within individual runs. The number of runs is determined by the number of random seeds (10 in this example). Since testing can be a lengthy process, we are providing a simple implementation of the callback mechanism for tracking its execution (line 4). The last line of Listing 1 demonstrates how to apply the prepared experiment on the 50words dataset from the UCR Time Series Classification Archive [3].

Listing 1. Implementing repeated cross-validation

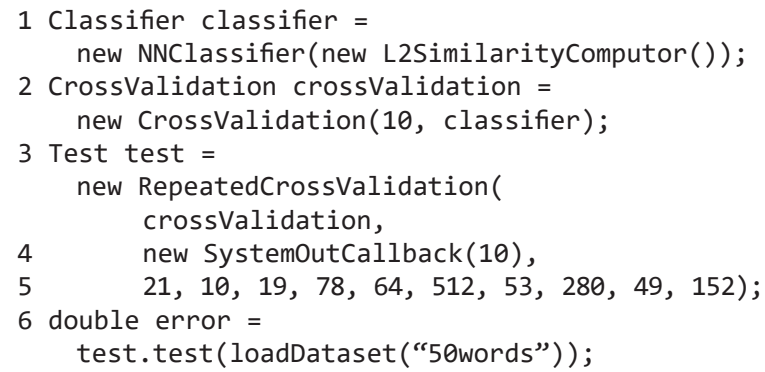




\section{Figure 5}

Fundamental interfaces of FAP: (a) SimilarityComputor; (b) PreprocessingTransformation; (c) Classifier; (d) Test; (e) TimeSeriesRepresentation; (f) Callback; (g) CallbackEnabled; (h) Resumable

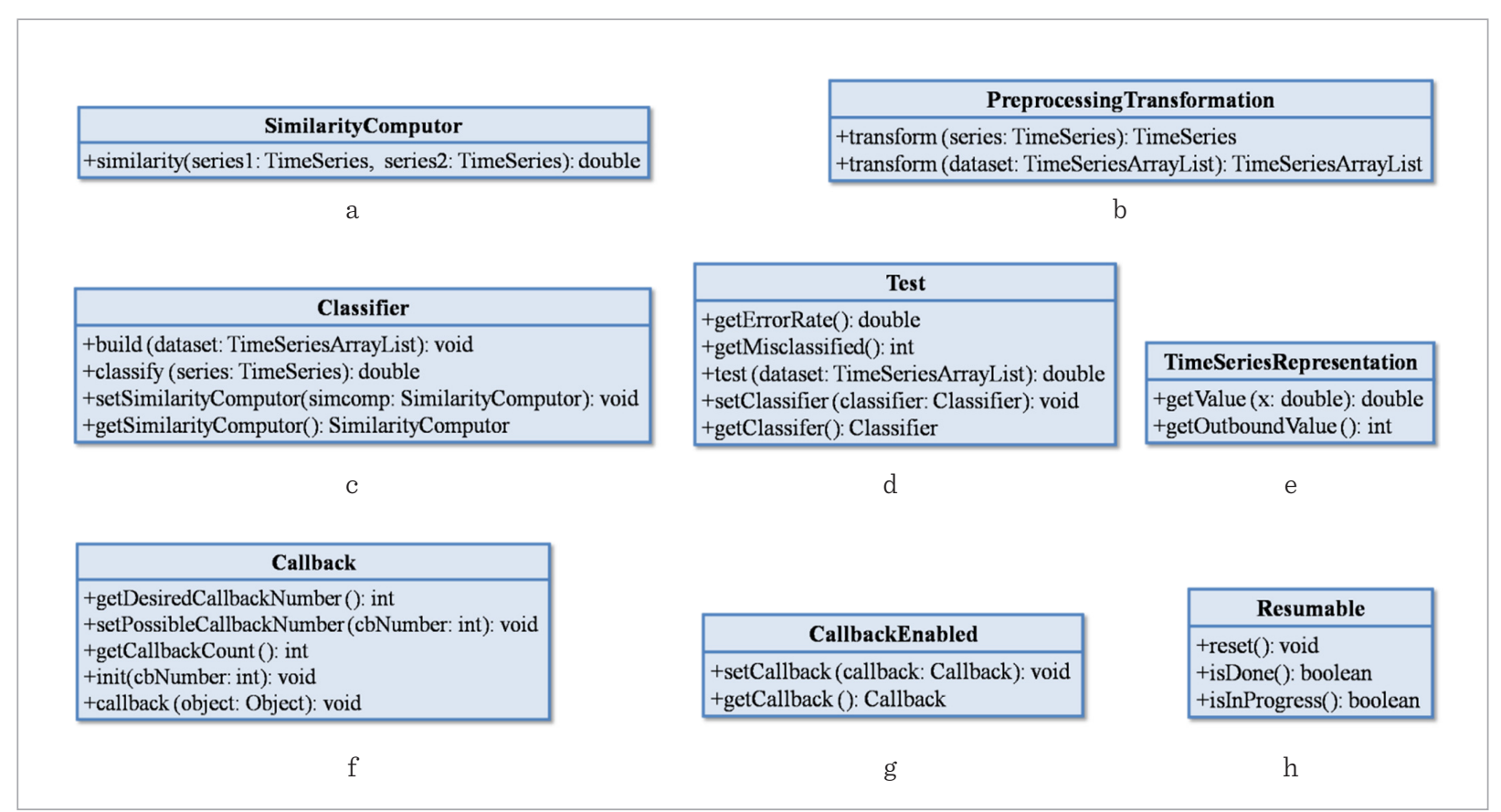

\section{Applications of FAP in Education}

In order to test FAP's functionalities more thoroughly and detect some potentially hidden errors, we recognized that it would be convenient to utilize FAP for educational purposes, too. In addition to that, employing FAP in education could point out the need for some new characteristic or for upgrading the existing ones. FAP is used in master's level subject "Artificial Intelligence 2" and in "Data Mining" seminar in Bachelor studies of Computer science with the aim of facilitating students' acquaintance with different tasks of time-series data mining. It has proved to be a very useful tool within doctoral studies, as well.

Application 1. SCVGUI. In the first stage of the development of FAP, we focused on implementing the most common time-series distance measures (like Euclidean distance, DTW, LCS, ERP, and EDR). In order to validate the correctness of our implementation [20], a dedicated Java application, called SCVGUI (Stratified Cross-Validation GUI), was created (Fig. 6a) within a seminar paper in doctoral studies. The input of this program is an experiment specification with the following structure: the first line contains the name of the experiment

\section{Figure 6}

Graphical User Interface for validating the implementations of common distance measures: (a) SCVGUI; (b) an experiment specification

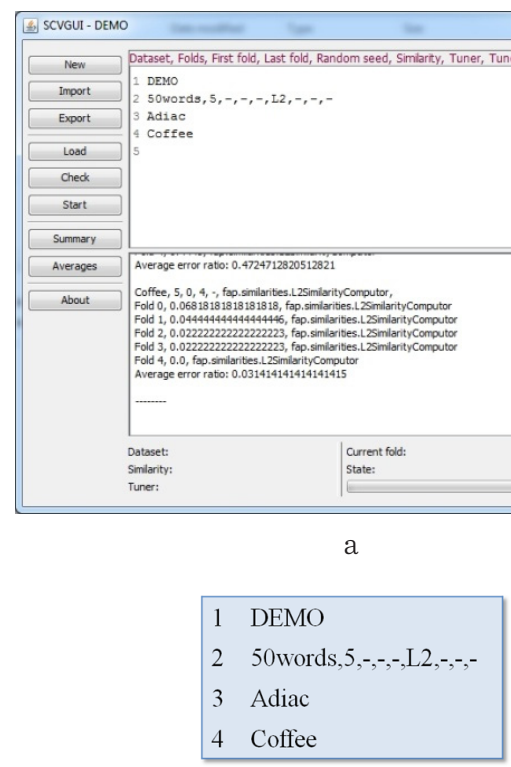

b 
and every subsequent line is reserved for an instruction that describes how to perform a given cross-validation evaluation using FAP in accordance with the algorithm described in [6] (SCVGUI can be extended with custom algorithms, too). In the current state of development, only the $1 \mathrm{NN}$ classifier is supported.

Fig. $6 \mathrm{~b}$ shows an example of SCVGUI experiment specifications. The name of this experiment is DEMO and it contains three instructions. The first one defines a 5-fold cross-validation using the 50words dataset from the UCR Time Series Classification Archive [3] and the Euclidean distance measure (L2). The other two instructions use the same parameters as the first one, so it is sufficient to provide only the names of the datasets: Adiac and Coffee. As the result, SCVGUI displays the error rates of the individual folds and their average value.

Owing to the fact that the design of FAP library supports tracking, interrupting and resuming long-running processes, SCVGUI allows the continuation of terminated experiments by serializing the corresponding Java object. The frequency of the serialization can be defined at the level of individual instructions. If it is omitted (as in case of the presented DEMO experiment), a default value (20) is used.

Application 2. DMGUI. To speed up some of our research efforts, another dedicated Java application was developed (within a seminar paper in doctoral studies, similarly as the in case of SCVGUI). The aim of the DMGUI (Distance Matrix GUI - Fig. 7a) program is to provide a flexible and easy-to-use interface for calculating distance matrices relying on the services of our FAP library. Similarly as in the case of SCVGUI, the input for this application is a specification of one or more commands for generating distance matrices. The first line of the specification is reserved for its name. Each of the following lines represents a command for generating a single distance matrix (or just a part of a matrix).

An example of DMGUI program input specification is presented in Fig. $7 \mathrm{~b}$. The name of this specification, which contains descriptions of three commands for generating distance matrices, is DEMO. The first command will generate a (lower) triangular matrix of distances between the time series of the 50words dataset using the DTW similarity measure constrained with the Sakoe-Chiba band (SCDTW). The width of the warping window is set to $25 \%$ of the time series' length. The other two commands use the same attributes as the first one, therefore only the names of the datasets (Adiac and Coffee) must be provided.

To further speed up the experiments with the kNN classifier, apart from calculating distance matrices, DMGUI is enabled to produce matrices of nearest neighbors, too. The $i$-th row of such a matrix contains all the time series of the given dataset (except the $i$-th series) sorted by their distance from the $i$-th series: the first element of the row is the most similar and the last one is the least similar.

\section{Figure 7}

Graphical User Interface for generating distance matrices: (a) DMGUI; (b) a DMGUI program

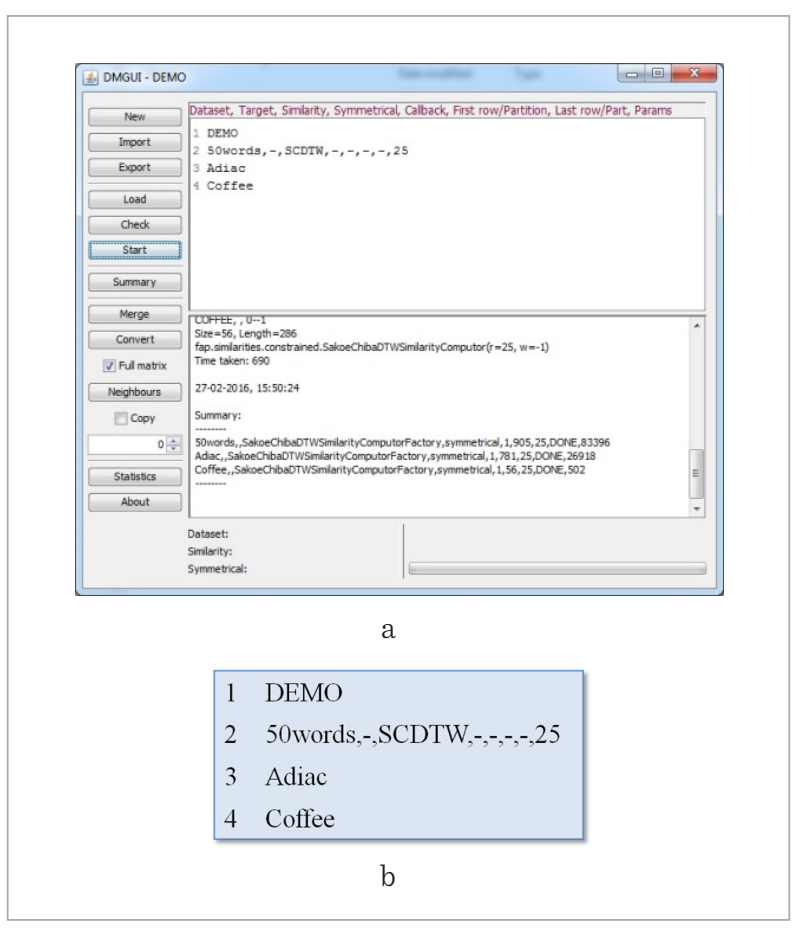

Relying on the ability of the FAP library to track and resume interrupted tasks, DMGUI supports stopping and continuing the process of generating distance matrices using object serialization. As in case of SCVGUI, the frequency of the serialization can be defined at the level of individual commands. If this parameter is omitted (like in the case of the provided example), a default value (20) is used. The core classes of this application were later employed in our agent-based distributed computing system for calculating distance matrices by utilizing networked computers [24]. 
Application 3. An extension of SCVGUI. As a part of a student paper in the Data Mining seminar (second and third year elective course, Bachelor studies of Computer science), aimed at familiarizing the students with the basic concepts of time-series data mining through utilizing the FAP library, the SCVGUI program was extended with the ability of directly setting the size of the Sakoe-Chiba band (rather than determining it using the tuning algorithm described in [6]). This extended version of the application was then used to investigate the influence of the Sakoe-Chiba band on the 1NN classifier in the case of the DTW distance measure by examining the classification accuracy for different warping window widths.

The experiments consisted of evaluating 10-fold stratified cross-validations using different warping window widths in range from $10 \%$ to $1 \%$ of the length of time series with (absolute) steps of 1 for several datasets from the UCR Time Series Classification Archive. The obtained smallest warping window widths which gave the best accuracy were compared with the values obtained in [21]. The results of the comparison are shown in Fig. 8.

\section{Figure 8}

Comparison of the results obtained with SCVGUI and with its extension

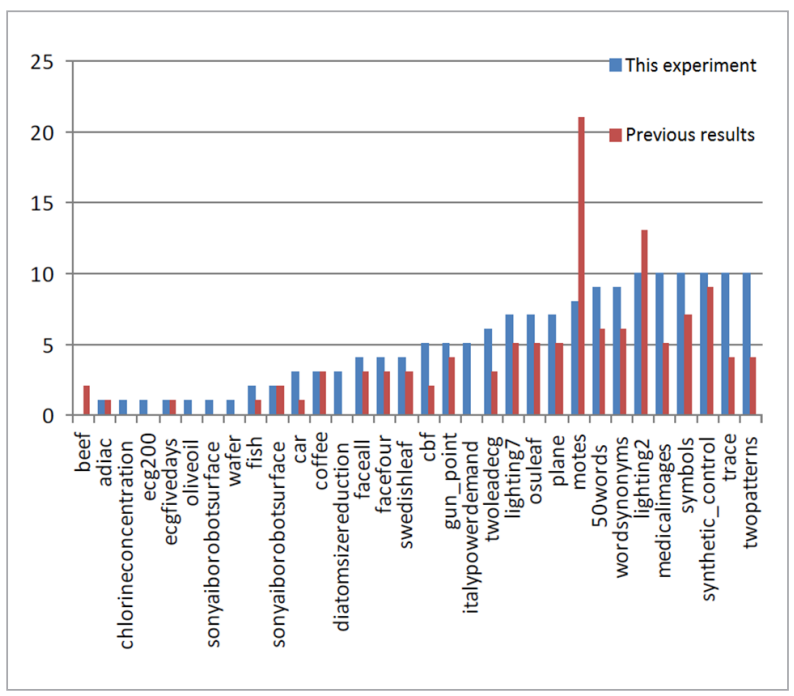

Application 4. Time-series reconstruction. Many tasks of machine learning and data mining are affected by the issues related to large datasets and high-dimensional data. These problems are known as the curse of dimensionality and they can deteriorate the performance of standard algorithms. In the field of time-series data mining, a common approach to overcome this phenomenon is to transform the data into a lower-dimensional representation while retaining the essential properties of the original series.

The topic of a seminar paper within doctoral studies was to investigate which representations are the most suitable for reconstructing the original time series and to find those distance measures that adapt well to the reconstructed data [17]. The analysis encompassed 85 datasets from the UCR Time Series Classification Archive [3] in combination with several time-series representations (DFT, DWT, PAA, Indexible PLA and SAX) in four different dimensions $(4,6$, 8 and 10) and a number of distance measures $\left(L_{1 / 2}, L_{1}\right.$, $\mathrm{L}_{2}, \mathrm{~L}_{\infty}$, unconstrained DTW, LCS, EDR and ERP).

In order to determine to what extent the reconstructed time series differ from the original ones, the reconstruction accuracy was calculated as the root-mean-square-deviation (RMSD), i.e. the Euclidean distance divided by the square root of time series dimensionality. The effectiveness of distance measures is calculated in a similar manner: as the RMSD between the distances of the original and the reconstructed series (denoted as SMRE - Similarity Measure Reconstruction Error). The averaged values of RMSD and SMRE are presented in Fig. 9 and Fig. 10, respectively. The analysis of the obtained results has shown that the most unstable time-series representation is DFT. Furthermore, while the elastic distance measures are less adaptive to DFT reconstruction than $\mathrm{L}_{\mathrm{p}}$ norms, they produced better results for all the other representations.

\section{Figure 9}

Averaged RMSD values

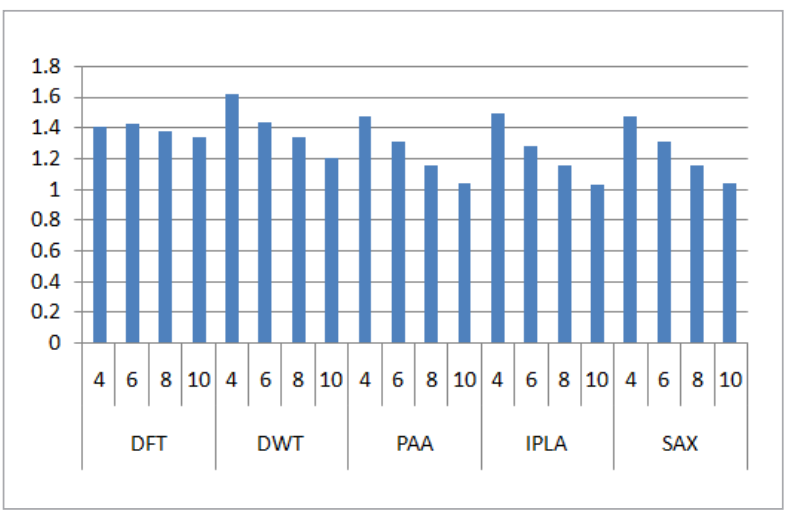


Figure 10

Averaged SMRE values

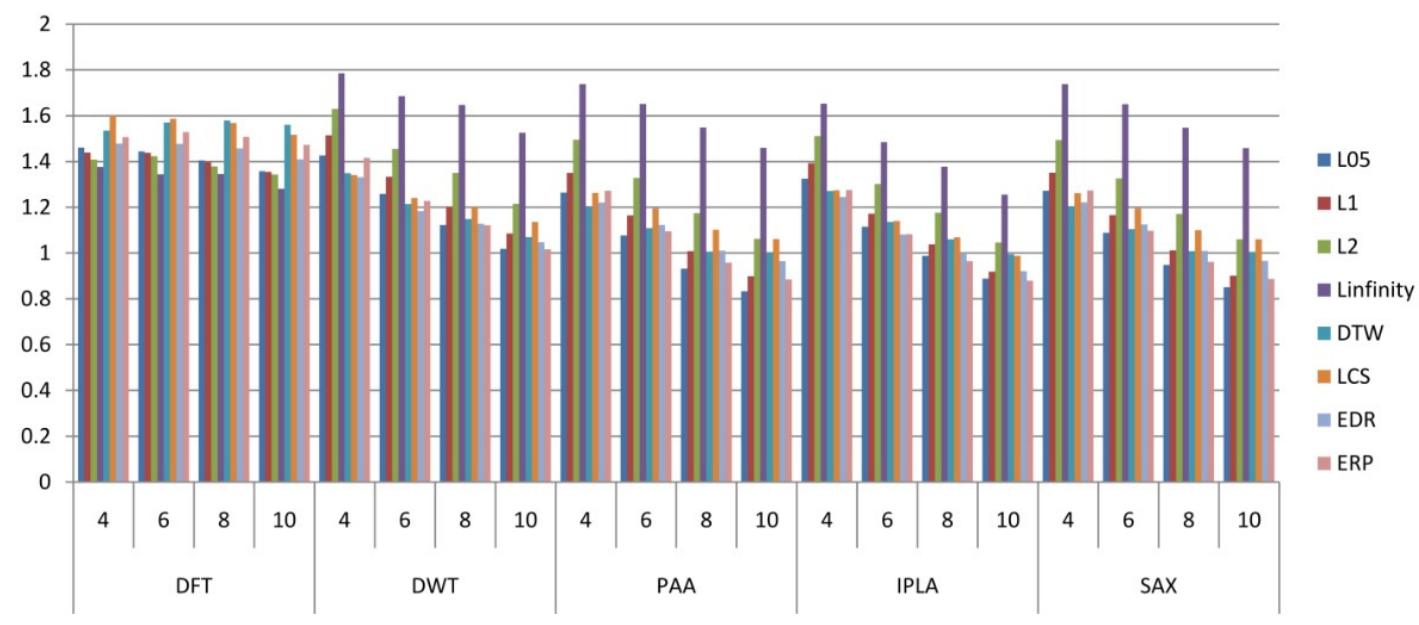

Even though some representation techniques existed in FAP before this application, during this project they were revised and optimized. In addition, several fundamental representation techniques were implemented, making FAP's repository of representations more comprehensive.

Application 5. Medical domain. Another student's seminar work and paper within the Data Mining course was performed in a specific medical area. The topic of this paper is analyzing medical records of pa- tients with HIV infection using different similarity measures by FAP. Two classes of PI/NRTI (protease inhibitors / non-nucleoside reverse transcriptase inhibitor) therapies were applied on patients and four kinds of recovery indicators were inspected (number of CD3, CD4, and CD8 T cells and the CD4/CD8 ratio).

The examined data were extracted from the database of the Infectious Diseases Clinic (Clinical Centre of Vojvodina) containing medical records of 136 patients with HIV infection (Fig. 11a). The data were

\section{Figure 11}

Analyzing medical records using FAP

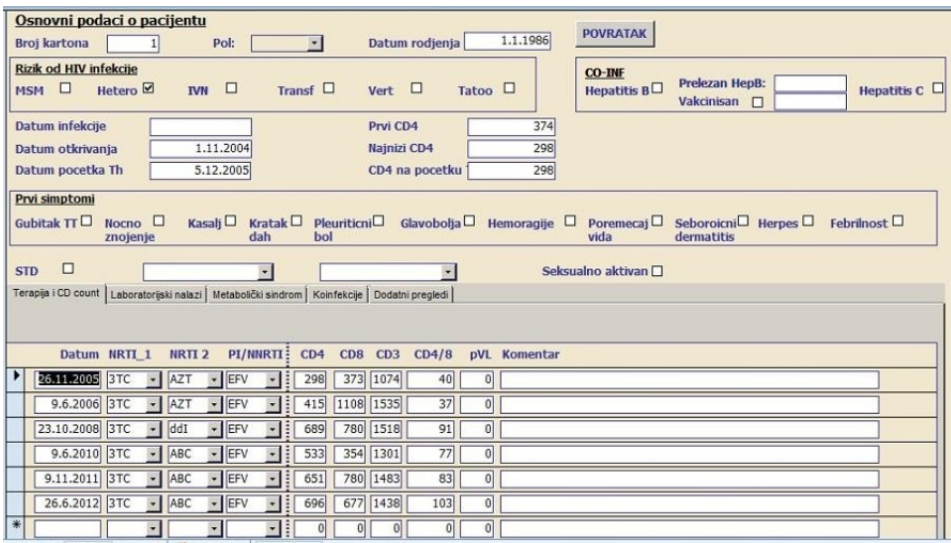

\begin{tabular}{|c|c|c|c|c|}
\hline & CD4 & CD8 & CD3 & CD4/CD8 \\
\hline EDR & 0,49648 & 0,480403 & 0,46819 & $\mathbf{0 , 4 3 0 3 2}$ \\
\hline LCS & 0,51756 & 0,44148 & $\mathbf{0 , 4 4 7 0 5}$ & 0,49334 \\
\hline DTW & 0,46565 & 0,45625 & 0,44967 & 0,44077 \\
\hline ERP & $\mathbf{0 , 4 1 5 0 8}$ & $\mathbf{0 , 4 2 7 4 8}$ & 0,45057 & 0,48232 \\
\hline
\end{tabular}


first transformed into time series to be evaluated with the FAP framework. After that, these time series were analyzed using the SCVGUI application.

Four time series were created for every patient, one for each recovery indicator. The time series were labeled based on the type of the applied therapy and placed in the appropriate dataset (CD3, CD4, CD8, or CD4/CD8). The obtained datasets were analyzed by applying three different stratified cross-validation experiments using SCVGUI: 2-fold, 5-fold, and 10-fold. The average values of the acquired error rates are presented in Fig. 11b.

The experiments were performed using the 1NN classifier in combination with the unconstrained versions of the four most commonly used elastic distance measures (DTW, LCS, EDR, and ERP). The objective of this experimental setup was to explore to what extent the extracted time series depend on the applied therapy and to identify the most appropriate distance measure.

Application 6. Financial domain. The sixth example of using FAP in an educational setting is a seminar paper that was done within the course Artificial Intelligence 2. This work investigates the possibility of using techniques of time-series analysis in predicting the change of stock prices based on one-year history. The raw data (stock price changes of the Nikola Tesla Airport in the period from 18 June 2014 to 17 June 2015 - Fig. 12) were downloaded from the Belgrade Stock Exchange's website (http://www.belex.rs).

Using the sliding window algorithm, the raw time series were split into three sets of subseries of the same length. The obtained subseries were labeled 1, 0 or -1 , depending on whether the price increased, remained the same or decreased. The labels of the $n$-th $(\mathrm{n}=1,2,3)$

\section{Figure 12}

Stock price changes in the form of time series

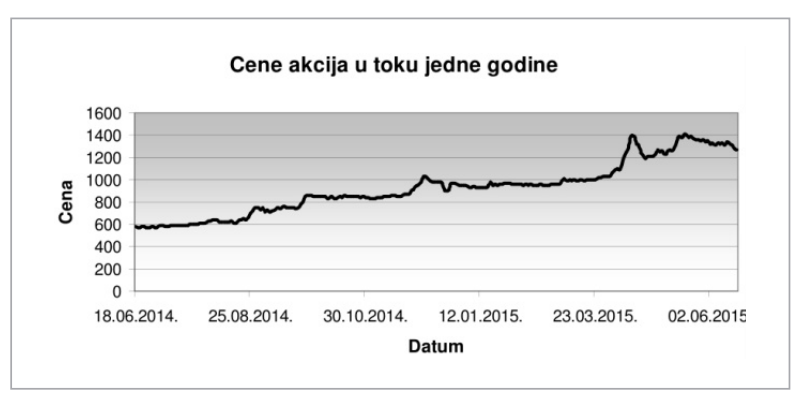

set were determined based on stock price changes $n$ days in advance. The obtained datasets were analyzed using SCVGUI by applying three different stratified cross-validation experiments using SCVGUI (2-fold, 10-fold, and LOO) and the following distance measures: $\mathrm{L}_{1}, \mathrm{~L}_{2}$, DTW, and ERP.

Application 7. Psychological domain. The last example is devoted to a master's thesis [8], developed intensively using FAP, and it is related to research papers [16] and [18]. The subject of this thesis is analyzing log file data obtained from SAM experiments using the FAP framework in order to find the best distance measure.

Three types of time series were extracted from the raw data: the first type describes the distance of the object from the starting point, the second type represents information about acceleration, and the third one specifies the deviations from the ideal trajectory (the shortest possible path). In [16], by applying hierarchical clustering on distance matrices (generated for these time series using the DTW, EDR and ERP distance measure) the ERP measure was selected as the most appropriate candidate to distinguish between the two types of navigators (i.e. "fast" and "accurate" navigators). An example of the clustering for the ERP measure is presented in Fig. 13 (taken from [16]).

Due to the fact that the results in [16] were not good enough to draw reliable reliable conclusions, additional examinations were needed. These extended experiments were conducted within this thesis [8]. In the first step, time series were labeled based on the type of the navigator they belong to (1 - "fast", 2 - "accurate"). After that, the datasets were analyzed with SCVGUI using 2-fold, 5-fold and 10-fold stratified cross-validation and the DTW, EDR, and ERP measures.

The tracks in the SAM experiments are of different length and complexity. Furthermore, various navigators complete the same track in different times. Therefore, the extracted time series are not of the same length. In the second phase of the experiments, the time series were scaled using linear interpolation. Using the time series prepared in this manner, the experiments were repeated with the following distance measures: DTW, LCS, EDR, ERP, $\mathrm{L}_{1}, \mathrm{~L}_{2}, \mathrm{~L}_{1 / 2}$, and $\mathrm{L}_{\infty}$.

The results showed that that different distance measures are best suited for different types of time series (distance, acceleration, deviation), but DTW generally gives the best overall outcomes, regardless of whether the time series are scaled or not. 


\section{Figure 13}

A dendrogram for hierarchical clustering for an ERP distance matrix

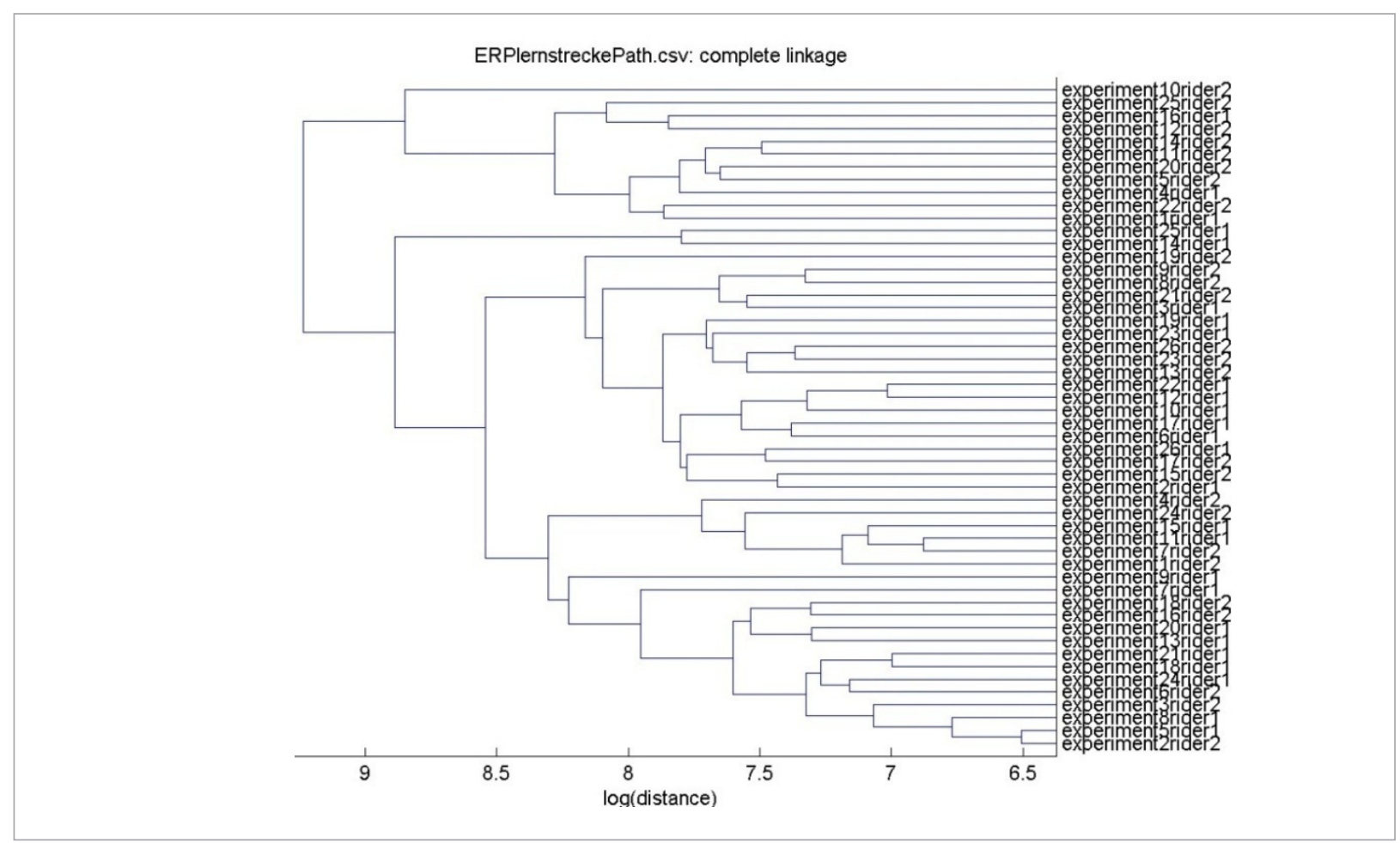

\section{Conclusion}

In this paper, we have described our Framework for Analysis and Prediction in which we intend to incorporate all main concepts of time-series data mining and analysis, like similarity measures, representations, pre-processing, classification, methods for evaluating the performance of classifiers and other functionalities. Furthermore, we have presented its application as an auxiliary tool in teaching computer science at all three levels of university education (bachelor, master's and doctoral studies).

\section{References}

1. Aggarwal, C. C. Data Mining: The Textbook. Springer Publishing Company, Incorporated, 2015. https://doi. org/10.1007/978-3-319-14142-8

2. Box, G. E. P., Jenkins, G. M., Reinsel, G. C., Ljung, G. M. Time Series Analysis: Forecasting and Control. 5th Edition, John Wiley \& Sons, Inc., Hoboken, New Jersey, 2015. https://doi.org/10.1111/jtsa.12194
Since the study program of Informatics at the Department of Mathematics and Informatics, Faculty of Sciences, University of Novi Sad, offers elective seminars and courses related to machine learning and data mining, and since the students, as part of their obligations, have to realize practical projects, FAP has proved to be an effective and suitable assisting tool. In addition, the students can choose to utilize our framework in carrying out experiments for the purpose of their undergraduate and master theses in different domains. Our positive experiences encourage us to believe that FAP can be useful for educational purposes in many other institutions as well.

3. Chen, Y., Keogh, E., Hu, B., Begum, N., Bagnall, A., Mueen, A., Batista, G. The UCR Time Series Classification Archive. 2015. http://www.cs.ucr.edu/ eamonn/ time_series_data/

4. Das, G., Gunopulos, D. Time Series Similarity and Indexing. In: Ye, N. (Ed.), The Handbook of Data Mining, Lawrence Erlbaum Associates, Mahwah, N. J., 2003, 279-304. 
5. Deza, M. M., Deza, E. Encyclopedia of Distances, 2nd Edition. Springer, Berlin, Heidelberg, 2013. https://doi. org/10.1007/978-3-642-30958-8

6. Ding, H., Trajcevski, G., Scheuermann, P., Wang, X., Keogh, E. Querying and Mining of Time Series Data: Experimental Comparison of Representations and Distance Measures. Proceedings of the VLDB Endowment, 2008, 1(2), 1542-1552. https://doi.org/10.14778/1454159.1454226

7. Esling, P., Agon, C. Time-Series Data Mining. ACM Computing Surveys, 2012, 45(1), Article No. 12. https://doi. org/10.1145/2379r776.2379788

8. Fodor, L. Analiza i identifikacija pogodnih mera sličnosti podataka vremenskih serija psiholoških eksperimenata. University of Novi Sad, Serbia, 2013.

9. Geler, Z. Role of Similarity Measures in Time Series Analysis, University of Novi Sad, Serbia, 2015.

10. Giusti, R., Batista, G. E. A. An Empirical Comparison of Dissimilarity Measures for Time Series Classification. 2013 Brazilian Conference on Intelligent Systems (BRACIS), Fortaleza, Brazil, 2013, 82-88. https://doi. org/10.1109/BRACIS.2013.22

11. Grossmann, W., Rinderle-Ma, S. Data Mining for Temporal Data. In Fundamentals of Business Intelligence. Springer, Berlin, Heidelberg, 2015, 207-244. https://doi. org/10.1007/978-3-662-46531-8_6

12. Han, J., Kamber, M., Pei, J. Data Mining: Concepts and Techniques, 3rd Edition. Elsevier, 2012. https://doi. org/10.1007/978-1-4419-1428-6_3752

13. Keogh, E., Chakrabarti, K., Pazzani, M., Mehrotra, S. Locally Adaptive Dimensionality Reduction for Indexing Large Time Series Databases. In Proceedings of the 2001 ACM SIGMOD International Conference on Management of Data, ACM, New York, NY, USA, 2001, 151-162. https://doi.org/10.1145/376284.375680

14. Keogh, E., Ratanamahatana, C. A. Exact Indexing of Dynamic Time Warping. Knowledge and Information Systems, 2005, 7(3), 358-386. https://doi.org/10.1007/ s10115-004-0154-9

15. Kirchgässner, G., Wolters, J., Hassler, U. Introduction to Modern Time Series Analysis, 2nd Edition. Springer, Berlin, Heidelberg, 2013. https://doi.org/10.1007/978-3642-33436-8

16. Kurbalija, V., von Bernstorff, C., Burkhard, H. D., Nachtwei, J., Ivanović, M., Fodor, L. Time-Series Mining in a Psychological Domain. Proceedings of the Fifth Balkan Conference in Informatics (BCI'12), ACM Press, New York, New York, USA, 2012, 58-63. https://doi. org/10.1145/2371316.2371328

17. Kurbalija, V., Bratić, B. Time Series Reconstruction Analysis. 2016 IEEE 8th International Conference on Intelligent Systems (IS), IEEE, 2016, 771-777. https://doi. org/10.1109/IS.2016.7737400
18. Kurbalija, V., Ivanović, M., von Bernstorff, C., Nachtwei, J., Burkhard, H. D. Matching Observed with Empirical Reality-What You See Is What You Get? Fundamenta Informaticae, 2014, 129(1-2), 133-147. https://doi.org/10.3233/ FI-2014-965

19. Kurbalija, V., Ivanović, M., Budimac, Z. Case-Based Curve Behaviour Prediction. Software: Practice and Experience, 2009, 39(1), 81-103. https://doi.org/10.1002/spe.891

20. Kurbalija, V., Radovanović, M., Geler, Z., Ivanović, M. A Framework for Time-Series Analysis. In Dicheva, D., Dochev, D. (Eds.), Artificial Intelligence: Methodology, Systems, and Applications. AIMSA 2010. Lecture Notes in Computer Science. Springer, Berlin, Heidelberg, 2010, 6304, 42-51. https://doi.org/10.1007/978-3-642-15431-7_5

21. Kurbalija, V., Radovanović, M., Geler, Z., Ivanović, M. The Influence of Global Constraints on Similarity Measures for Time-Series Databases. Knowledge-Based Systems, 2014, 56, 49-67. https://doi.org/10.1016/j.knosys.2013.10.021

22. Laxman, S., Sastry, P. S. A Survey of Temporal Data Mining. Sadhana, 2006, 31(2), 173-198. https://doi. org/10.1007/BF02719780

23. Marteau, P. F. Time Warp Edit Distance with Stiffness Adjustment for Time Series Matching. IEEE Transactions on Pattern Analysis and Machine Intelligence, 2009, 31(2), 306-318. https://doi.org/10.1109/TPAMI.2008.76

24. Mitrović, D., Ivanović, M., Geler, Z. Agent-Based Distributed Computing for Dynamic Networks. Information Technology and Control, 2014, 43(1), 88-97. https://doi. org/10.5755/j01.itc.43.1.4588

25. Mitsa, T. Temporal Data Mining. Taylor \& Francis, 2010. https://doi.org/10.1201/9781420089776

26. Serrà, J., Arcos, J. L. An Empirical Evaluation of Similarity Measures for Time Series Classification. Knowledge-Based Systems, 2014, 67, 305-314. https://doi. org/10.1016/j.knosys.2014.04.035

27. Wang, X., Mueen, A., Ding, H., Trajcevski, G., Scheuermann, P., Keogh, E. Experimental Comparison of Representation Methods and Distance Measures for Time Series Data. Data Mining and Knowledge Discovery, Springer US, 2013, 26(2), 275-309. https://doi. org/10.1007/s10618-012-0250-5

28. Yin, H., Yang, S., Ma, S., Liu, F., Chen, Z. A Novel Parallel Scheme for Fast Similarity Search in Large Time Series. China Communications, 2015, 12(2), 129-140. https://doi. org/10.1109/CC.2015.7084408

29. Zaki, M. J., Meira, W. Jr. Data Mining and Analysis: Fundamental Concepts and Algorithms, Cambridge University Press, New York, 2014.

30. Zhang, Z., Tang, P., Duan, R. Dynamic Time Warping Under Pointwise Shape Context. Information Sciences, 2015, 315, 88-101. https://doi.org/10.1016/j.ins.2015.04.007 